\title{
Calibration of a monochromator using a lambdameter
}

\author{
T. Schwarzmaier, A. Baumgartner, P. Gege, K. Lenhard \\ Deutsches Zentrum für Luft- und Raumfahrt (DLR), Institut für Methodik der Fernerkundung, \\ 82234 Oberpfaffenhofen, Germany
}

\begin{abstract}
The standard procedure for wavelength calibration of monochromators in the visible and near infrared wavelength range uses low-pressure gas discharge lamps with spectrally well-known emission lines as primary wavelength standard. The calibration of a monochromator in the wavelength range of 350 to $2500 \mathrm{~nm}$ usually takes some days due to the huge number of single measurements necessary. The useable emission lines are not for all purposes sufficiently dense and at the appropriate wavelengths. To get faster results for freely selectable wavelengths, a new method for monochromator characterization was tested. It is based on measurements with a lambdameter taken at equidistant angles distributed over the grating's entire angular range. This method provides a very accurate calibration and needs only about two hours of measuring time.
\end{abstract}

Keywords: Monochromator, wavelength-calibration, lambdameter, wavemeter

\section{INTRODUCTION}

The German Aerospace Center (DLR) operates the Calibration Home Base (CHB) as a facility for the calibration of airborne imaging spectrometers and field spectrometers in the wavelength range from 350 to $2500 \mathrm{~nm}$ [1]. For spectral measurements, an Oriel MS257 ${ }^{\mathrm{TM}} 1 / 4 \mathrm{~m}$ monochromator illuminated by a quartz tungsten halogen lamp (QTH) is used as light source, which has a specified wavelength accuracy of $0.15 \mathrm{~nm}$. For accurate calibration results, precise knowledge of the monochromator characteristics is essential. So far, gas discharge lamps are used to determine the spectral properties. However, a full wavelength calibration takes some days due to the huge number of single measurements necessary, and is nevertheless based on few emission lines with fixed wavelengths.

In order to reduce the calibration effort and to improve the coverage of reference wavelengths, an alternate calibration method was developed and tested using a spare monochromator Mc-Pherson Model 2051 with high light throughput and good wavelength accuracy. The outdated manual control was equipped with a new stepper motor with PC interface to allow computer control. Due to the complex mechanics that connects the motor with the grating, the relationship between motor position and monochromator wavelength is an unknown nonlinear function. To set a specific wavelength, a mathematical equation is needed which describes this relationship and makes it possible to calculate the motor position for each wavelength. The standard measurements with gas emission lines did not yield satisfactory results, as there were not enough useful wavelengths for an accurate polynomial fit. To get more values with sufficient accuracy, a new calibration method, which uses a lambdameter, was developed.

\section{THE STANDARD CALIBRATION PROCEDURE}

The standard procedure for wavelength characterization uses low-pressure gas discharge lamps with spectrally wellknown emission lines as primary standard (Figure 1A). Instead of the QTH lamp a gas discharge lamp (1) is installed in front of the monochromator's entrance slit. A radiometer is mounted in front of the exit slit (8). For alignment, the grating (5) is turned into the zero order position where it works as a mirror. In this position the lamp (1) is adjusted to provide maximum radiometer signal. For calibration, the monochromator's grating is rotated in small steps around the expected angle corresponding to the center wavelength of the selected emission line, and measurements of the relative light intensity are taken with the radiometer (Figure 2). 
By fitting a Gaussian curve through these intensity values, the angular position of the grating can be calculated for each spectral line. These measurements are repeated for several spectral lines. After calibration is finished, the QTH lamp must be reassembled and adjusted to maximum radiometer signal to get it in the same position as the discharge lamps.

With this method, highly accurate results are obtained, but the lamps must be exchanged, each lamp aligned, and hundreds of measurements undertaken. If the function relating grating position and wavelength is unknown, a polynomial fit to the wavelength values is necessary. The amount of available emission lines is small for an accurate polynomial fit.

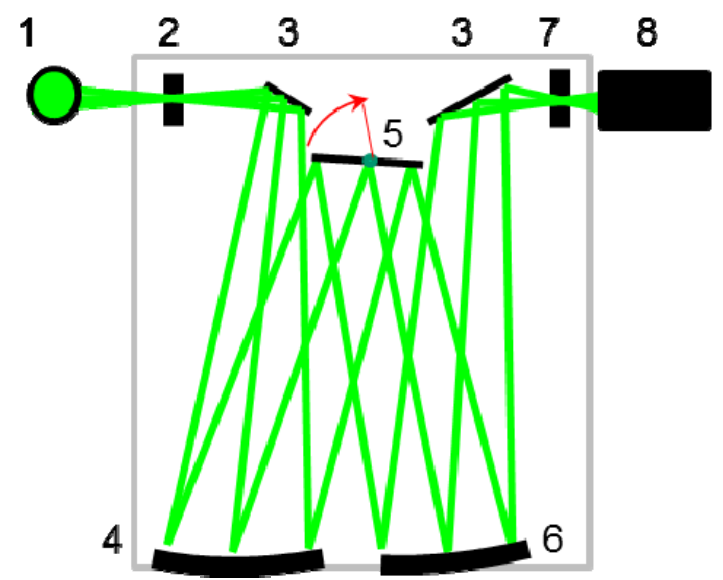

A: Setup for calibration using gas discharge lamps

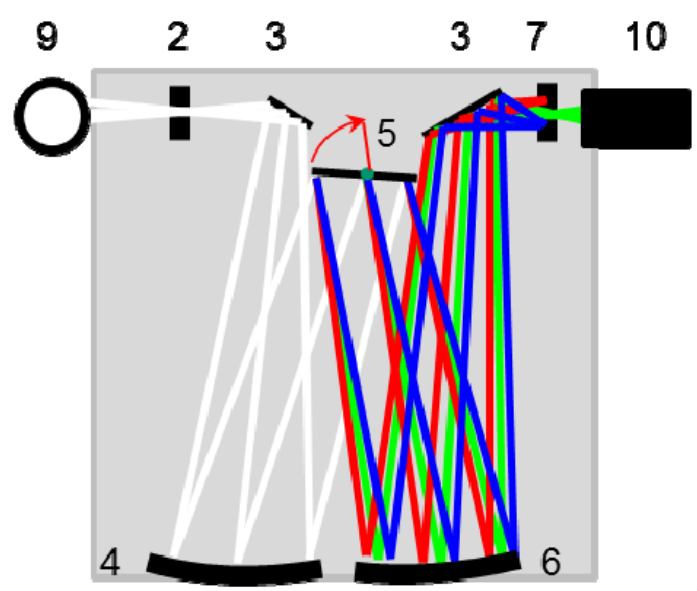

B: Setup for calibration using a lambdameter

Figure 1: Monochromator setup for calibration: (1) gas discharge lamp, (2) entrance slit, (3) folding mirrors, (4) collimating mirror, (5) turnable grating, (6) focussing mirror, (7) exit slit, (8) radiometer, (9) QTH lamp, (10) Lambdameter

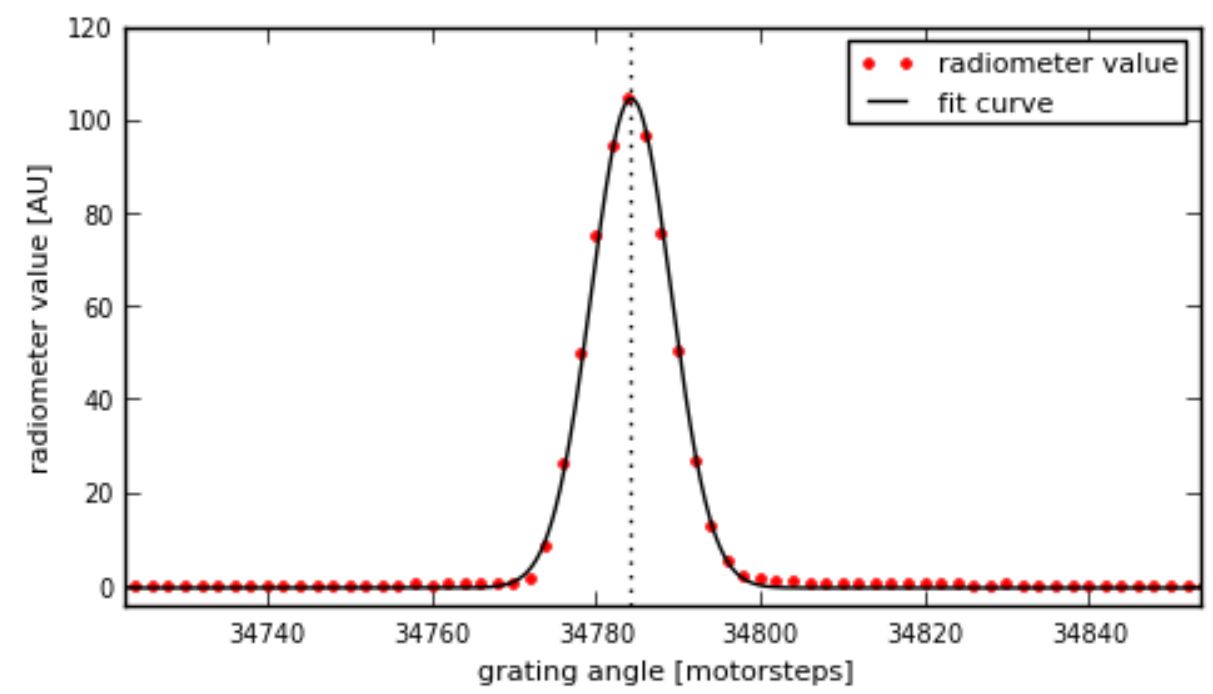

Figure 2: Example of the radiometer measurements to get the motor position of one gas emission line 


\section{CALIBRATION USING A LAMBDAMETER}

\subsection{MEASUREMENT SETUP}

The monochromator setup is shown in Figure 1B. The monochromator "MC-Pherson Model 2051" is illuminated, as usual, by a $100 \mathrm{~W}$ QTH lamp (9) in a lamp housing with focusing optics. Its light is dispersed by a grating (5) with 600 grooves $/ \mathrm{mm}$ and a blaze wavelength of $1850 \mathrm{~nm}$. A stepper motor driven by a positioning control (Nanotec SMCI33) is used to turn the grating and to set the wavelength at the exit slit (7). For calibration, the optical fiber of a lambdameter (10) was fixed in front of the monochromator exit slit.

Lambdameters, also called wavemeters, are instruments designed to measure single spectral lines with a very high accuracy. The used model "GWU Lambda Scan" is an Échelle-spectrometer with a spectral accuracy of $5 \times 10^{-5}(+/-$ $0.0125 \mathrm{~nm} @ 500 \mathrm{~nm}$ ), that covers a wavelength range from 240 to $1250 \mathrm{~nm}$ [2]. It is calibrated at CHB using the spectral line of an Nd:YAG-Laser.

The output signal of the monochromator has multiple peaks at different wavelengths due to different diffraction orders. Since the lambdameter provides only one wavelength value, it selects the wavelength corresponding to the strongest peak. The wavelengths of the other orders can be calculated using the grating equation.

\subsection{MEASUREMENTS}

The stepper motor can be adjusted in steps from 0 to 52000. To characterize the entire range, the grating was positioned in intervals of 200 motor steps in the complete range. At each position 20 single measurements were taken with the lambdameter and averaged to one wavelength value. In this way, 260 measurements were taken.

The uncertainty of the obtained wavelengths was changing across the motor positions due to varying intensity of the measurement signal. In order to avoid inaccurate data, the lambdameter software automatically excludes values with low signal to noise ratio. Due to this procedure no values remained from 0 to 8000 motor steps and some gaps were obtained in the range from 30000 to 50000 motor steps, but the remaining 135 values cover the biggest part of the range (Figure $3)$.

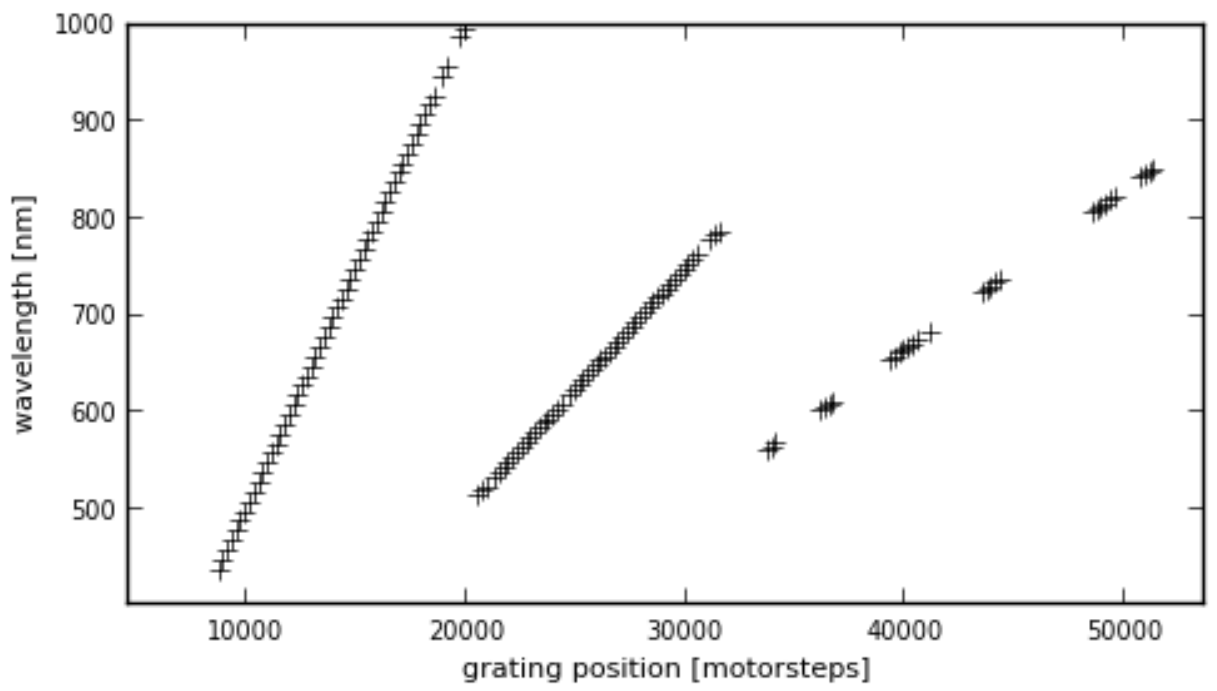

Figure 3: Wavelength values at different grating positions taken with the lambdameter 


\subsection{DATA PROCESSING}

The monochromator provides signals at several diffraction orders. The lambdameter automatically selects the strongest of these signals and determines the center wavelength of the measured peak. Consequently, the measurements are in the first instance mixtures from different diffraction orders. Here, the number of diffraction orders is three (Figure 3). Further processing requires sorting the data according to the diffraction order, i.e. to produce a continuous curve. For that purpose, the values in the left and right part of Figure 3 are converted to the same order like the middle part. The wavelengths of the left part from 8000 to 20000 motor steps were divided by two; the wavelengths of the right part from 33000 to 52000 motor steps were multiplied by 1.5. This leads to a continuous curve covering a wavelength range from 200 to $1250 \mathrm{~nm}$ (Figure 4). It is suited to determine the required relationship between motor position and wavelength by fitting a polynomial to the values.

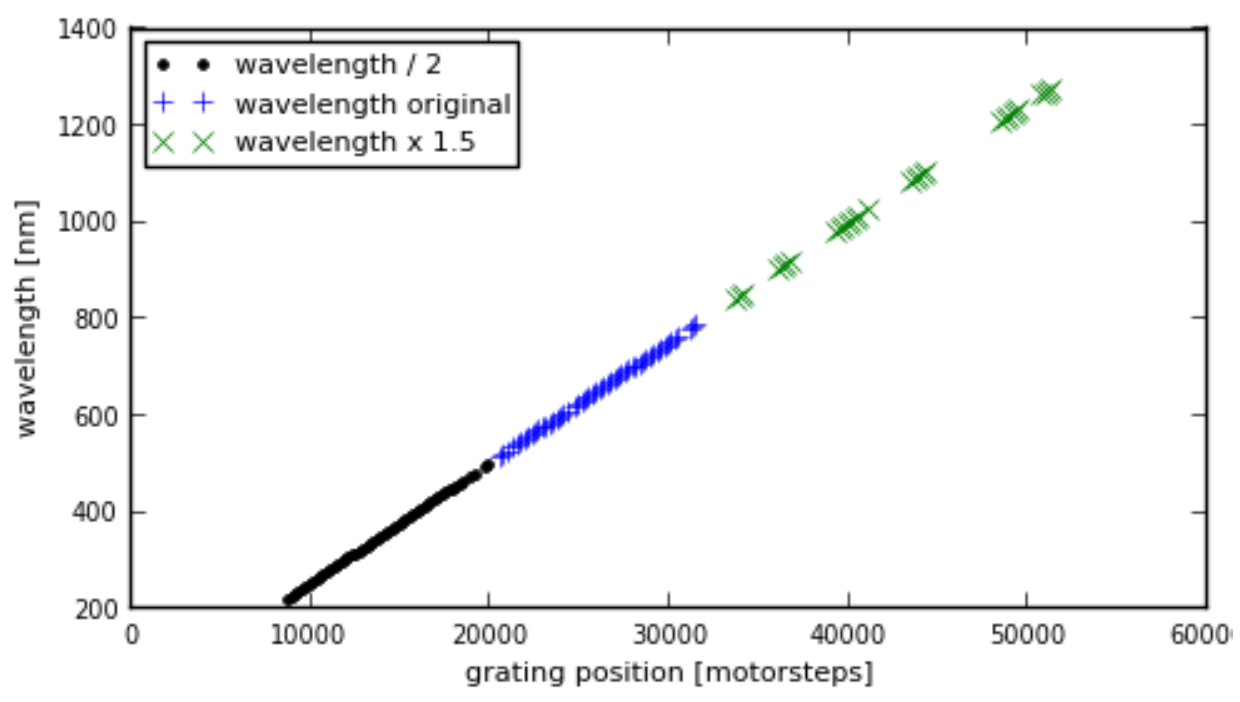

Figure 4: Data from Figure 3sorted for diffraction order.

\subsection{DETERMINING AN APPROPRIATE POLYNOMIAL DEGREE}

To find out which polynomial degree suits best to the data, degrees from 1 to 9 were tested. After fitting, the wavelength for each value was calculated using the polynomial. To compare the results, the average of the absolute differences between measured and calculated wavelength values were plotted (Figure 5). This plot shows that the fit gets rapidly better until the fourth degree, and then improves only slightly. For the calibration of the monochromator the sixth degree was chosen. For this relationship, the average of absolute differences is $0.005 \mathrm{~nm}$, and the standard deviation is 0.007 nm.

To illustrate the quality of the calibration equation for all motor positions, the differences of each measured wavelength to the calculated wavelength are plotted in Figure 6. Adding the standard deviation of $0.007 \mathrm{~nm}$ to the uncertainty of the lambdameter calibration $\left(\Delta \lambda / \lambda=5 \times 10^{-5}\right)$ using the propagation of uncertainty, the following uncertainties for wavelength calibration of the monochromator are obtained: 0.0188 nm @350 nm; 0.0505 nm @ 1000 nm; 0.1252 nm @ $2500 \mathrm{~nm}$. 


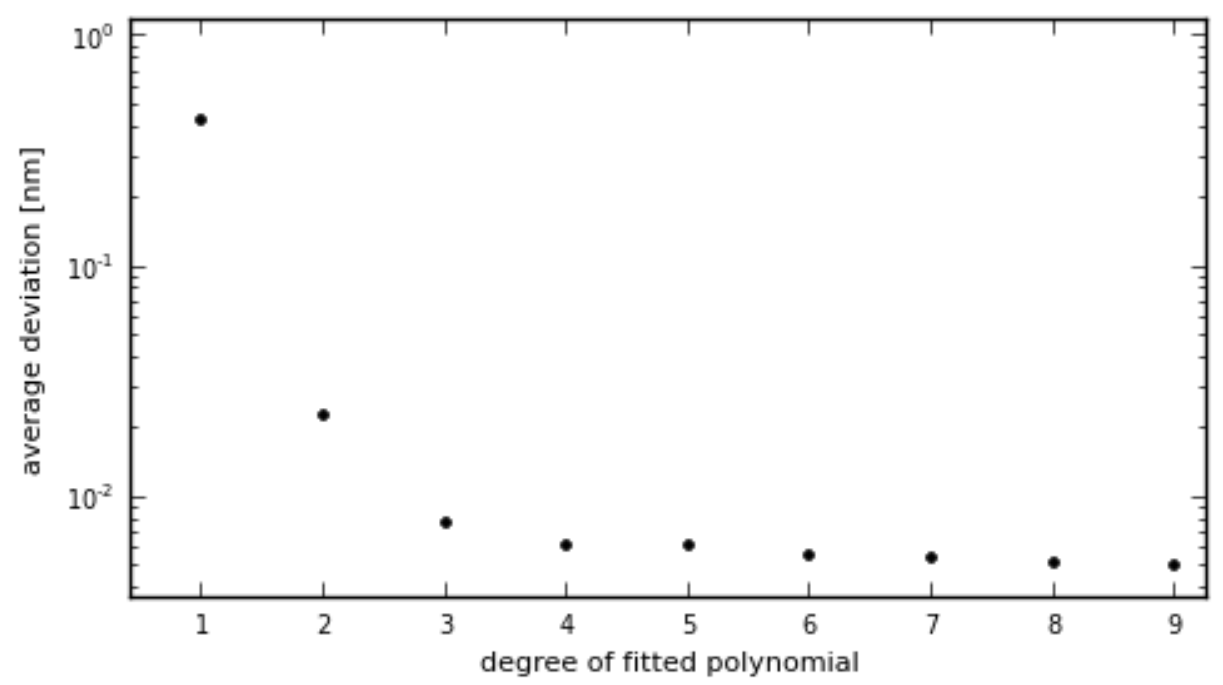

Figure 5: Average absolute difference between measured wavelength and polynomial fit for different polynomial degrees.

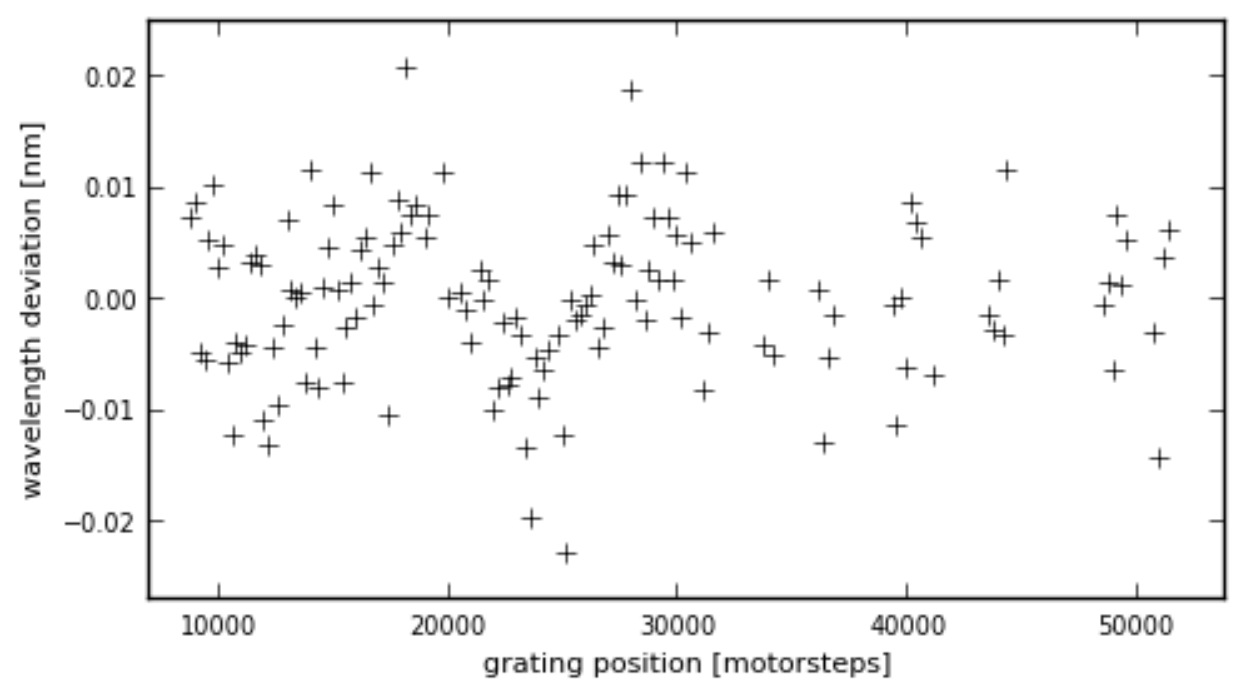

Figure 6: Differences between measured wavelengths and wavelengths from obtained calibration equation.

\subsection{VALIDATION OF THE WAVELENGTH RANGE}

In chapter 3.3 it was assumed that the monochromator provides signal in more diffraction orders than observed with the lambdameter. To verify this assumption, measurements were made similar to the measurements described in chapter 3.1, but with a spectrometer type "SVC hr1024i" instead of the lambdameter at position (10) of Figure 1B. The measurements with the spectrometer don't require high wavelength calibration accuracy, as they are only used to determine the usable diffraction orders.

The spectrometer took data in the range of 350 to $2500 \mathrm{~nm}$ in intervals of 200 motor steps in the complete range. The three strongest signals of each measurement were selected. Signals less than 100 Digital Numbers (DN) were omitted, thus some measurements have only one or two peaks. A Gaussian curve was fitted to the values of each peak to find the center wavelengths. The center wavelengths were plotted versus the respective motor positions (Figure 7). 
This plot shows that the monochromator covers a wavelength range from $380 \mathrm{~nm}$ to $2500 \mathrm{~nm}$. There also might be weak signals from $350 \mathrm{~nm}$ to $380 \mathrm{~nm}$ (data not shown), but the spectrometer is not sufficiently sensitive to verify this. The wavelength range is entirely covered by one diffraction order, which has twice the wavelength of the one calculated in chapter 3.3 Three diffraction orders are visible that differ by factors of 1/2, 1/3, and 1/4 from the wavelengths of the continuous order, but the last one has only weak signals.

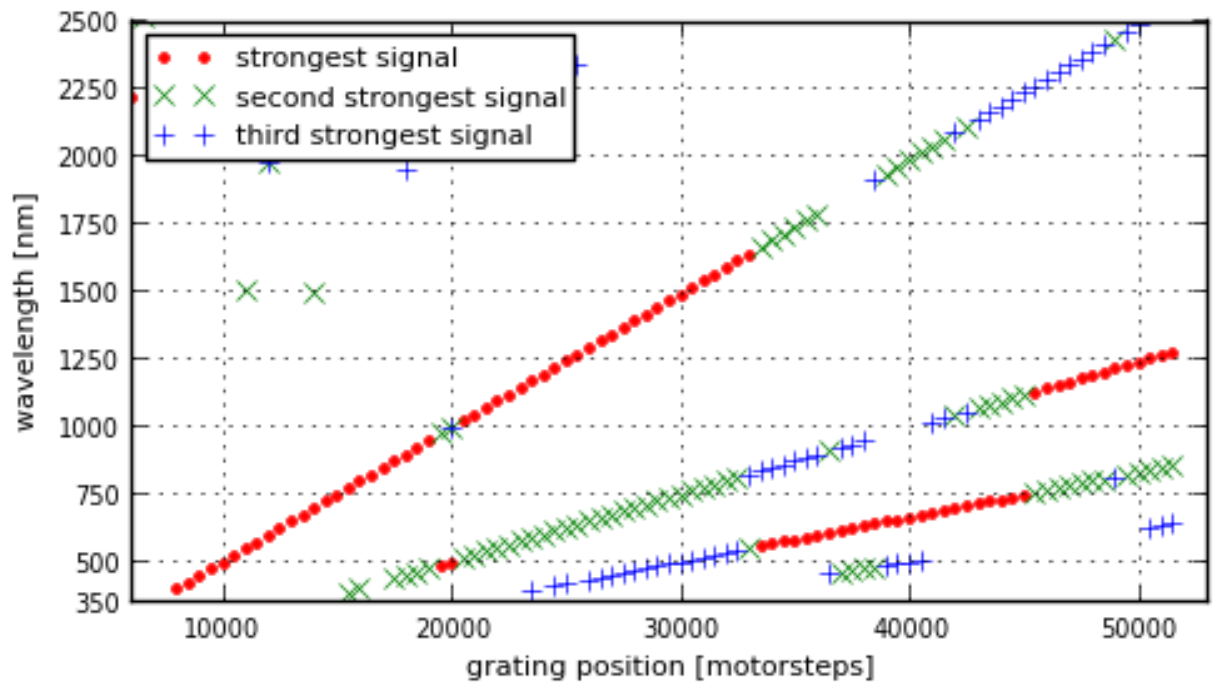

Figure 7: Diffraction orders of the monochromator measured with a spectrometer.

\section{CONCLUSION}

The developed method of monochromator calibration in the wavelength range from 350 to 2500 nm is a fast and accurate alternative to the standard procedure. Because of the good wavelength coverage it is a useful method for the characterization of unknown monochromators. However, a prerequisite is a well-calibrated, wavelength stable lambdameter, because unlike the conventional method, the measurements are not related to a primary standard directly. The measurements show that a wavelength accuracy of $0.1 \mathrm{~nm}$ or better is possible with the used setup.

\section{REFERENCES}

[1] Gege, P., Fries, J., Haschberger, P., Schötz, P., Schwarzer, H., Strobl, P., Suhr, B., Ulbrich, G. \& Vreeling, J. (2009). Calibration facility for airborne imaging spectrometers. ISPRS Journal of Photogrammetry \& Remote Sensing 64, 387-397.

[2] GWU-Lasertechnik Vertriebsges.mbH, GWU LambdaScan-usb Technical Manual version 3.22 / GWU 